\title{
Solid Pseudopapillary Tumor of Pancreas: A Case Report
}

\author{
RASHID MM ${ }^{\mathrm{a}}$, RABBI H ${ }^{\mathrm{b}}$, ISLAM A ${ }^{\mathrm{c}}$, HUSAIN MM$^{\mathrm{c}}$, KABIR MM $^{\mathrm{d}}$, AHMED T AHM ${ }^{\mathrm{e}}$, \\ AKTAR KM ${ }^{\mathrm{e}}$, ALAM $\mathrm{H}^{\mathrm{e}}$, RAHMAN MA ${ }^{\mathrm{f}}$, ALI $\mathrm{M}^{\mathrm{g}}$
}

\begin{abstract}
Solid pseudopapillary tumor of pancreas is a rare tumor almost exclusively affecting the young female and accounts for $<1 \%$ of all pancreatic tumors. Most hypothesis suggests that the tumor arises from embryonic pleuripotent stem cells. Although mostly benign in nature, the tumor may show malignant potentials and the patient may present with hepatic, omental or other meatstasis. High index of suspicion is the key for early dianosis. Good quality imaging (USG and CT scan) \& FNAC are necessary for proper evaluation of the lesion.
\end{abstract}

\section{Case Report}

A 13-year old girl was admitted under the department of Hepato-Biliary-Pancreatic Surgery with complaints of left-sided abdominal pain and occasional vomiting for last one year. For last few months she noticed abdominal distension and a palpable mass on the left side of abdomen. Her pain was agonizing in nature, not associated with any

a. Dr. Md. Mamunur Rashid, Associate Professor, Dept. of HBPS, BIRDEM Hospital, Dhaka-1000, Bangladesh

b. Dr. Hashim Rabbi, Assistant Professor, Dept. of HBPS, BIRDEM Hospital, Dhaka-1000, Bangladesh

c. Dr. Azharul Islam, Dr. Muhd. Mustaque Husain, Assistant Professor, Dept. of Surgical Emergency, BIRDEM Hospital, Dhaka-1000, Bangladesh

d. Dr. Md. Mohsin Kabir, Assistant Professor, Dept. of GHPD, BIRDEM Hospital, Dhaka-1000, Bangladesh

e. Dr. A H M Tanvir Ahmed, Dr. K M Akhtar, Dr. Hasina Alam, Senior Medical Officer, Dept. of HBPS, BIRDEM Hospital, Dhaka-1000, Bangladesh

f. Dr. M Anisur Rahman, Professor, Dept. of GHPD, BIRDEM Hospital, Dhaka-1000, Bangladesh

g. Prof. Mohammad Ali, Professor, Dept. of HBPS, BIRDEM Hospital, Dhaka-1000, Bangladesh

Address of Correspondence to: Dr. Md. Mamunur Rashid, Associate Professor, Dept. of HBPS, BIRDEM Hospital, Dhaka1000, Bangladesh, E-mail: drmamunr@yahoo.com

Received : 09 February, 2012 Accepted : 12 October 2012
Peroperative frozen section biopsy may be necessary to ascertain its malignant potential. Radical resection is the best modality of treatment for achieving curative results and a better long-term survival. We are reporting our experience of treating a case of solid pseudopapillary tumor of pancreas in BIRDEM Hospital.

Keywards: Pseudopapillary tumor, Solid-cystic tumor, Distal Pancreatectomy

(Birdem Med J 2013; 3(1): 54-58)

precipitating or aggravating factors, occasionally followed by vomiting and usually relieved spontaneously after persisting for one to three days. She had no history of haematemesis, melaena or weight loss. Her bowel \& bladder habits were normal and the Immunization schedule was completed.

Clinically she was mildly anaemic and there was no lymphadanopathy. The abdominal mass was occupying the epigastric, left hypochondriac \& lumber regions, about $10 \mathrm{~cm}$ in diameter, surface was smooth, mildly tender, firm to hard in consistency, almost immobile and was not moving with respiration. Haematological and biochemical parameters were within normal limits except mild anaemia. Ultrasonography revealed a complex mass - $14 \mathrm{~cm} \times 13 \mathrm{~cm}$ in size - suggestive of a pancreatic pseuducyst. CT scan of abdomen showed a well defined mixed density mass lesion with significant cystic component at left hypochondriac \& upper lumber region $15 \mathrm{~cm} \times 10 \mathrm{~cm} \times 12 \mathrm{~cm}$ in size, causing superomedial displacement of head \& body of pancreas \& inferomedial displacement of left kidney. 
(A)

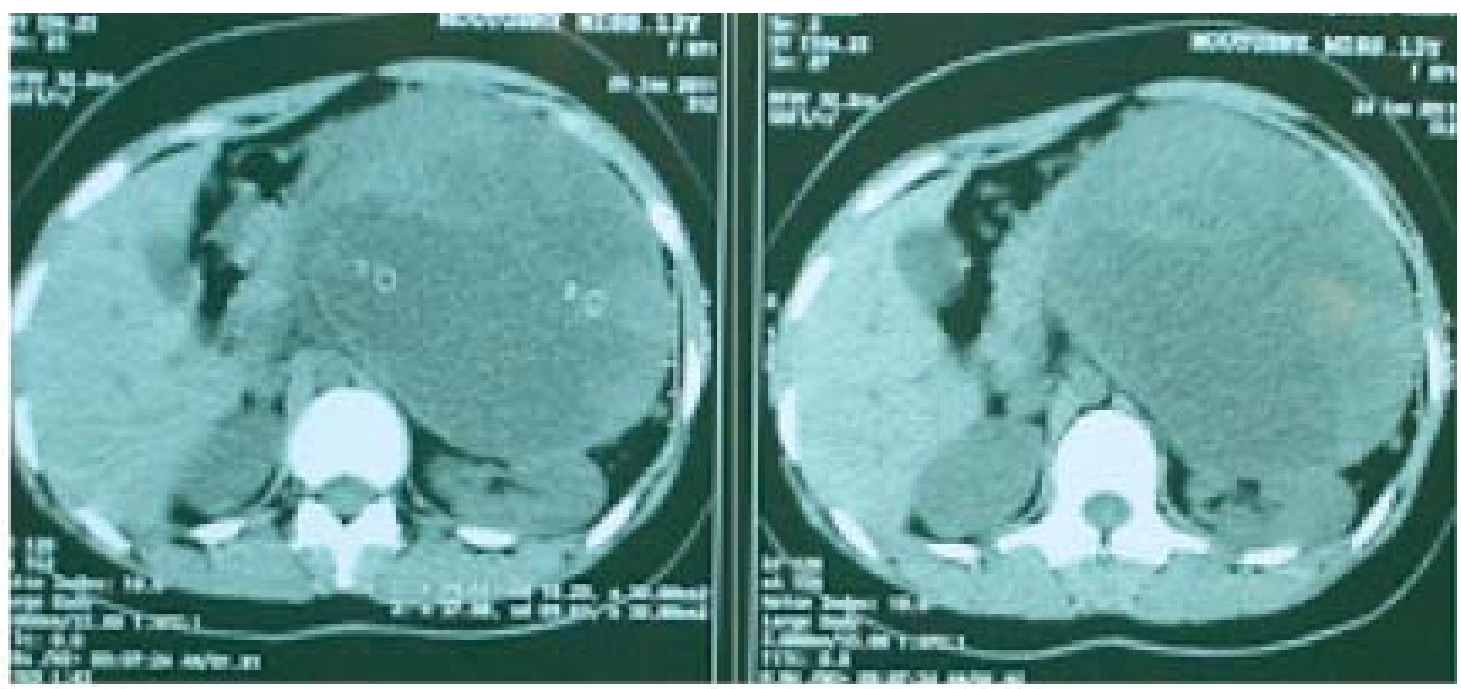

(B)

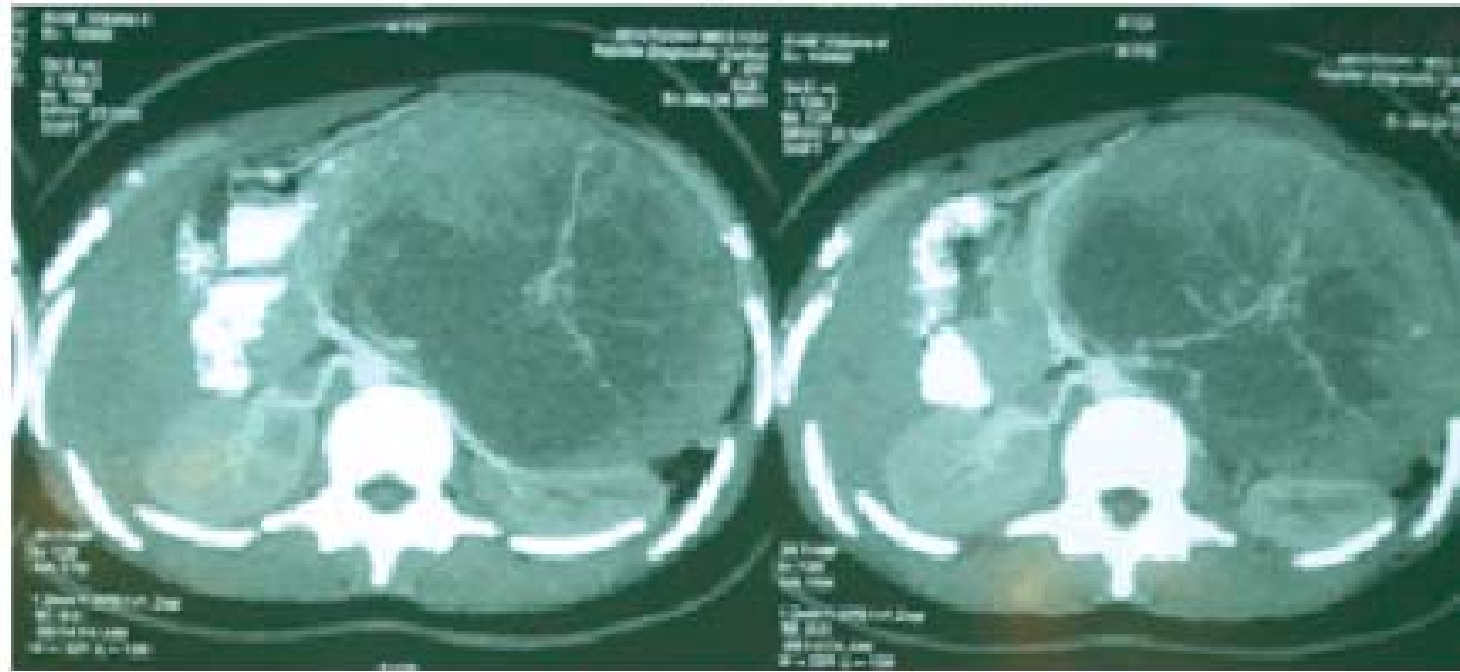

(C)

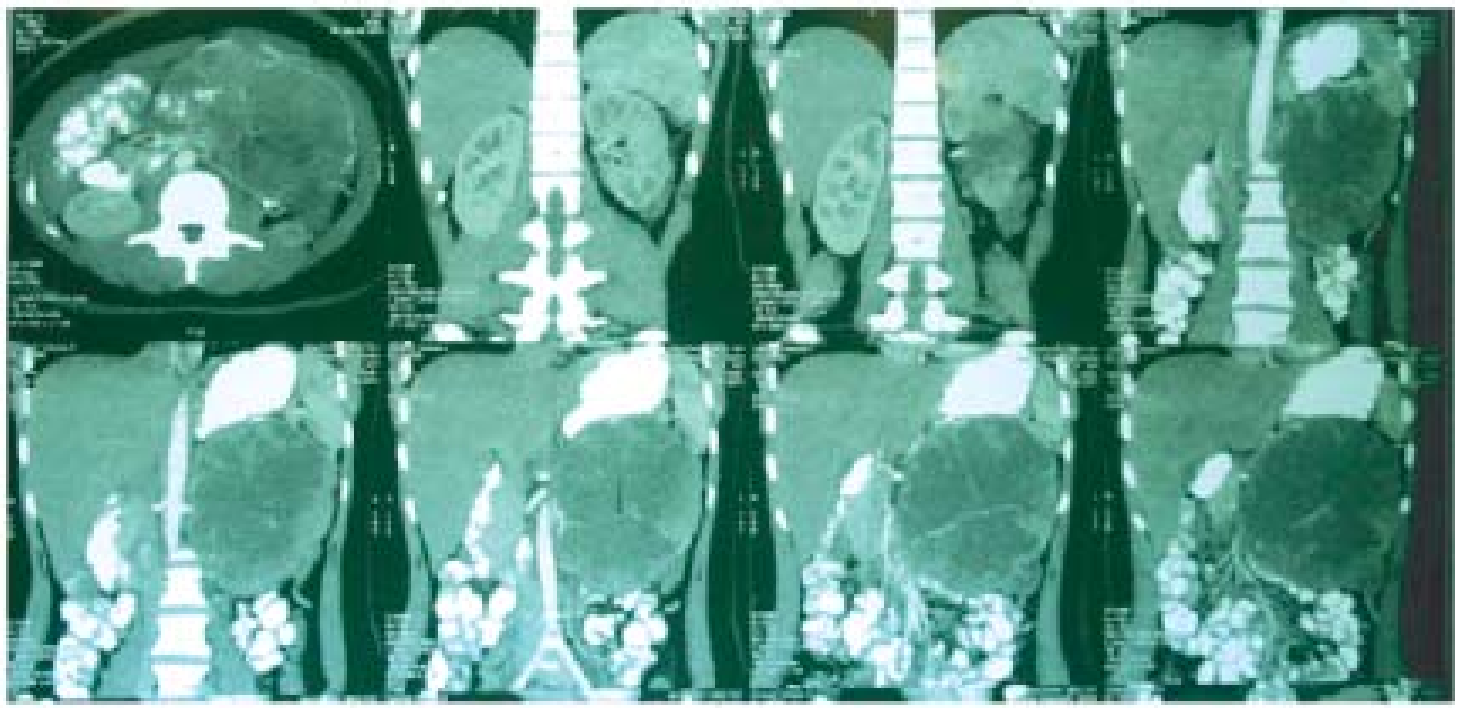

Fig.-1: $C T(A, B, C)$ appearance of the abdominal cyst 
After intravenous contrast it showed diffuse contrast enhancement of solid \& septal contrast enhancement. Impression was mucinous macrocystic adenoma of pancreas. The other possibilities are pancreatic cystadenoma, pancreatic cystadenocarcinoma, pancreatic pseudocyst and tumor of mesechymal origin (GIST). She was screened for malignancy by CA 19.9 \& CA 125, which were also normal. USG guided FNAC revealed polygonal cells having round to oval nuclei with indistinct nucleoli arranged in pseudopapillary form and the diagnosis was solid pseudopapillary neoplasm of pancreas.

The patient was prepared for en-mass resection of distal pancreas with splenectomy, if the intraoperative frozen section biopsy is positive for malignancy. She was vaccinated against pneumococcus, meningococcus \& haemophillus influenzae type $b$.

Under combined thoracic epidural and general anesthesia, laparotomy was done by supraumbilical transverse incision. There was a solid and cystic huge mass involving the middle and distal part of pancreas with multiple engorged vessels overlying the mass. The mass was adherent with transverse colon \& mesocolon and encased the splenic vessels. Enlarged lymph nodes were found at hepatoduodenal ligament. Other abdominal organs were apparently healthy. There was no metastatic deposit in the liver or elsewhere. En-mass resection of the pancreatic tumour was performed with splenectomy and hepatoduodenal lymphadenectomy.

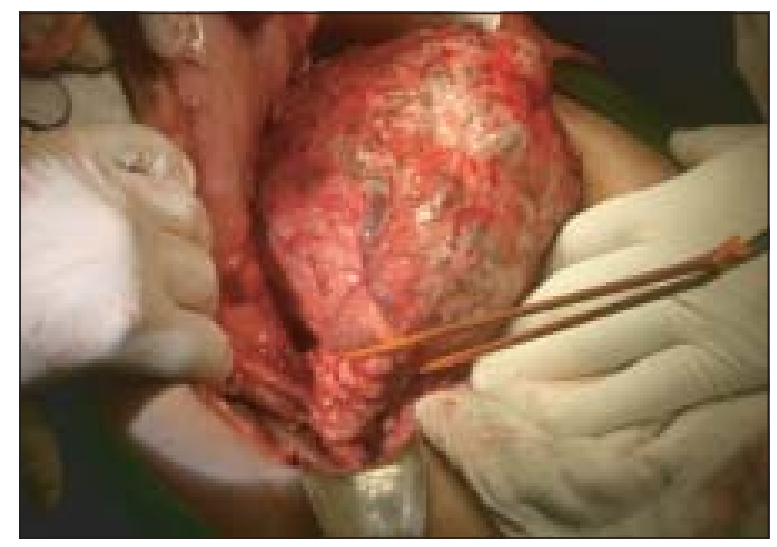

Fig.-2a: A sling is passed behind the junction of body \& neck\& Pancreas

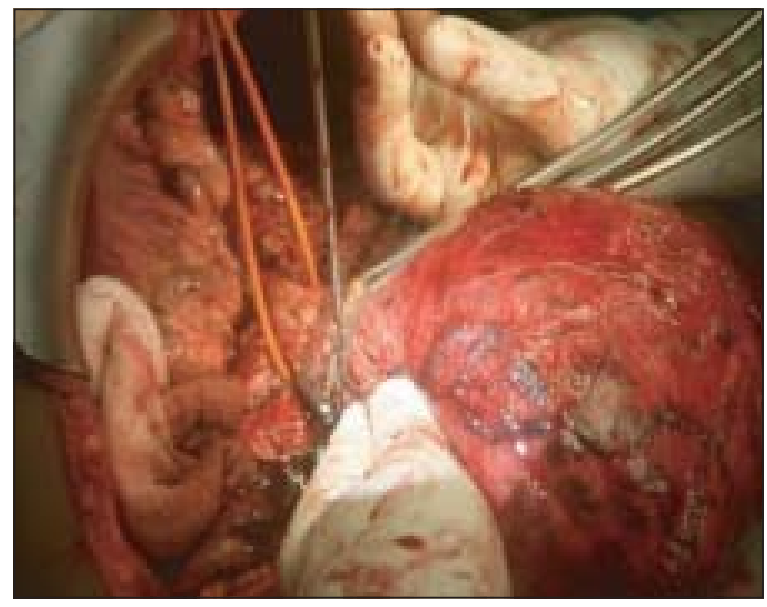

Fig.-2b: Resection of pancreatic tumour over the sling

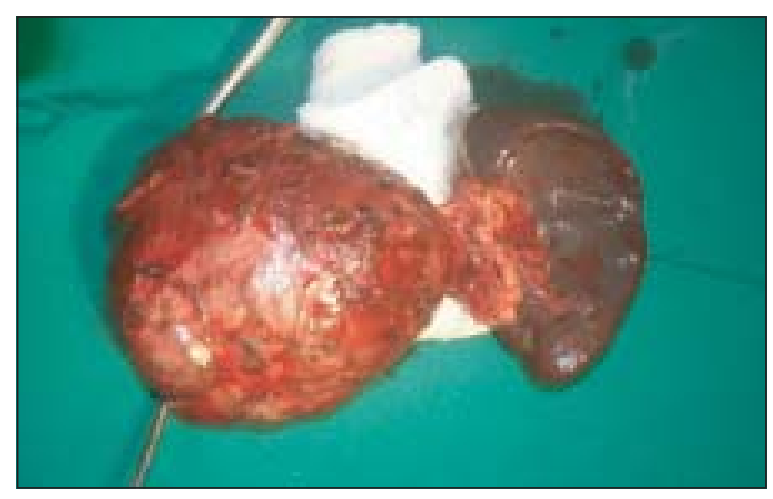

Fig.-3: Tumour removed en-mass with Spleen

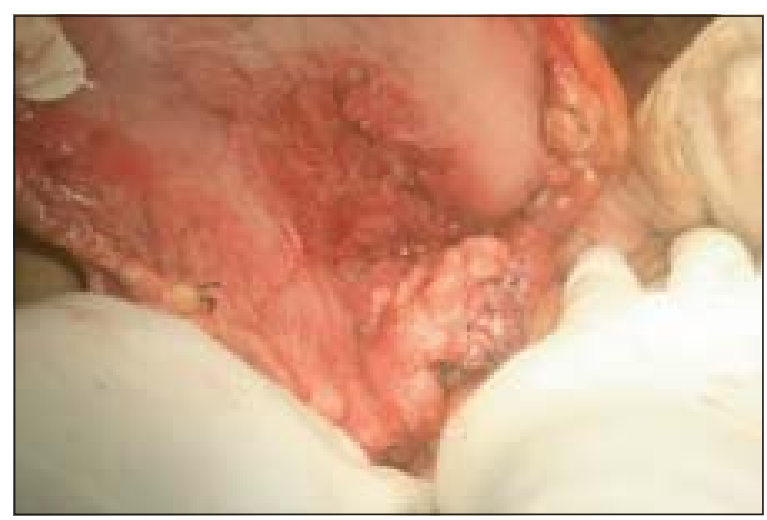

Fig.-4: Distal end of the pancreas closed

Postoperative period was uneventful except, minor wound infection. The wound swab culture isolated Acinetobacter infection sensitive to Colistin. It was managed by surgical debridement \& dressing and the antibiotics offered according to culture \& sensitivity of wound swab. Her glycaemic status was normal (5.7 - 
$7.4 \mathrm{mmol} / \mathrm{l}$ ) and platelet count raised upto 4,50,000/cmm, which later on declined to 3,30,000/cmm. Histopathology of the specimen confirmed solid pseudopapillary tumour (encapsulated) of pancreas with chronic nonspecific lymphadenitis, without any evidence of malignancy. Oral phenoxymethyl penicillin started from $10^{\text {th }}$ day after splenectomy. Secondary closure of the wound was done on $34^{\text {th }}$ POD. She was discharged with a follow up plan. Clinical \& Imaging: 3 monthly for $1^{\text {st }} \mathrm{yr}$ \& then yearly, periodic monitoring of endocrine as well as exocrine function and hematological assessment. Antibiotic prophylaxis is essential for her post splenectomy status.

\section{Discussion}

Solid pseudo papillary neoplasm is also known as Gruber-Frantz tumor ${ }^{1}$, Solid \& cystic tumor, Solid \& papillary neoplasm and Papillary epithelial neoplasm. It is a rare disease and is almost exclusively seen in young females and occurs in the second or third decades of life ${ }^{2,3,4}$. This accounts for $<1 \%$ of all pancreatic tumors, and most present a benign behavior ${ }^{5}$; but rarely may show low grade malignant potentials. ${ }^{6,7}$ Histochemical, immunohistochemical, and electron microscopic examinations showed polymorphic differentiation, which means that part of the tumor cells differentiated into duct epithelium, acinus, and endocrine cells (primary constituents of the pancreas). These findings suggest the hypothesis that the tumor originates from pleuripotential embryonic stem cells. ${ }^{4,5}$ For this the term pancreatic embryonic tumors seems preferable to papillary cystic and solid tumor of the pancreas to delineate the origin of the tumor and to reflect some of its biologic characteristics. ${ }^{5}$

It is equally distributed in the head, body \& tail of pancreas with or without calcification. Extra pancreatic location of this tumor (eg. liver) is uncommon. Synchronous metastatic lesions may be present in the liver \& peritoneum (15\%). ${ }^{8}$

Curative resection is ensured by complete excision, negative resection margin and absence of metastatic disease. When the tumor is malignant, recurrence may be even after ten years of curative resection. ${ }^{1}$ A local recurrence rate of $6.2 \%$ is reported in cases treated by radical surgical excision, and hepatic or Krukenbergtype distant metastases develop in $5.6 \%$ of cases. ${ }^{9}$ It has been reported that neoadjuvent chemotherapy has increased the rate of resectability of the tumor with metastasis ${ }^{10}$ and post operative radiotherapy may add survival benefit. ${ }^{11}$ However, most authors agree that aggressive surgical resection is the best modality of treatment for achieving curative results and a better long-term survival ${ }^{11,12,13}$.

Five years survival following primary resection is $95 \%$ if the tumor is confined to pancreas at presentation. ${ }^{11}$ Complete resection is associated with long time survival, even with documented metastases.

\section{Conclusion}

In a young female presenting with a pancreatic mass, a high index of clinical suspicion is key to diagnosis of solid pseudopapillary tumor of pancreas. FNAC may confirm it. Intraoperative frozen section biopsy is needed to evaluate a suspected metastatic lesion and plan the surgery. Radical resection with tumor free margin in absence of metastatic lesions offers a long time survival. Regular follow up is essential for early detection of early local recurrence and metastasis.

\section{References}

1. Frantz VK. Tumors of the pancreas, Atlas of Tumor Pathology, VII. In: Fascicles 27 and 28. Washington: Armed Forces Institute of Pathology, 1959 ;32-33.

2. Compagno J, Oertel JE, Kemzar M. Solid and papillary epithelial neoplasm of the pancreas, probably of smallduct origin: a clinico-pathological study of 52 cases (Abstract) Lab Invest. 1979;40:248-49.

3. Jagannath P, Bhansali MS, Murthy SK, Mohandas KM, Swaroop VS, DeSouza LJ. Solid and cystic papillary neoplasm of pancreas-a report of seven cases. Indian J Gastroenterol 1994;13:112-14.

4. Mao C, Guvendi M, Domenico DR, Kim K, Thomford NR, Howard JM. Papillary cystic and solid tumors of the pancreas: a pancreatic embryonic tumor? Studies of three cases and cumulative review of the world's literature. Surgery 1995;118:821-28.

5. Mao C, Guvendi M, Domenico D, et al. Papillary cystic and solid tumors of the pancreas: a pancreatic embryonic tumor? Studies of three cases and a cumulative review of the world's literature. Surgery 1995;118:821-28.

6. Klimstra DS, Wenig BM, Heffess CS. Solid pseudopapillary tumor of the pancreas: a typically cystic carcinoma of low malignant potential. Semin Diagn Pathol. 2000;17:66-88.

7. Nishihara K, Nagoshi M, Tsuneyoshi M, et al. Papillary cystic tumors of the pancreas: assessment of their malignant potential. Cancer. 1993;71:82-92. 
8. Ogawa T, Isaji S, Okamura K, et al. A case of radical resection for solid cystic tumor of the pancreas with widespread metastases in the liver and greater omentum. Am J Gastroenterol 1993;88:1436-39

9. Gonzalez-Campora R, Rios Martin JJ, Otal Salaverri C, Otal Salaverri C, Hevia Vazquez A, Valladolid JM, et al. Papillary cystic neoplasm of the pancreas with liver metastasis coexisting with thyroid papillary carcinoma. Arch Pathol Lab Med 1995;119:268-73

10. Vollmer Cm, Dixon E, and Dr Grant. Management of a solid pseudopapillary tumor of the pancreas with liver metastases. HPB (Oxford) 2003; 5(4): 264-67.
11. Yoon DY, Hines OJ, Bilchik AJ. Solid and papillary epithelial neoplasm of the pancreas: aggressive resection for cure. Am Surg 2001; 67:1195-99.

12. Horvath KD, Chabot JA. An aggressive resectional approach to cystic neoplasm of the pancreas. Am J Surg 1999; 178: 269-74.

13. Kaufman SL, Reddick RL, Stiegel M, Wild RE, Thomas CG., Jr Papillary cystic neoplasm of the pancreas: a curable pancreatic tumor. World J Surg 1986;10:851-59. 